PROCEEDINGS OF THE

AMERICAN MATHEMATICAL SOCIETY

Volume 134, Number 12, December 2006, Pages 3595-3603

S 0002-9939(06)08418-8

Article electronically published on June 8, 2006

\title{
RIGIDITY OF CERTAIN HOLOMORPHIC FOLIATIONS
}

\author{
DAVID MARÍN \\ (Communicated by Carmen C. Chicone)
}

\begin{abstract}
There is a well-known rigidity theorem of Y. Ilyashenko for (singular) holomorphic foliations in $\mathbb{C P}^{2}$ and also the extension given by Gómez-Mont and Ortíz-Bobadilla (1989). Here we present a different generalization of the result of Ilyashenko: some cohomological and (generic) dynamical conditions on a foliation $\mathcal{F}$ on a fibred complex surface imply the d-rigidity of $\mathcal{F}$, i.e. any topologically trivial deformation of $\mathcal{F}$ is also analytically trivial. We particularize this result to the case of ruled surfaces. In this context, the foliations not verifying the cohomological hypothesis above were completely classified in an earlier work by X. Gómez-Mont (1989). Hence we obtain a (generic) characterization of non-d-rigid foliations in ruled surfaces. We point out that the widest class of them are Riccati foliations.
\end{abstract}

\section{INTRODUCTION AND MAIN RESULTS}

This paper deals with the comparison of the analytical and topological classifications of holomorphic foliations. Roughly speaking, when two categorical contexts are considered, an object is called rigid if the weakest notion implies the stronger one. This is the situation for a wide class of (singular) holomorphic foliations on $\mathbb{C P}^{2}$ as was stated by $\mathrm{Y}$. Ilyashenko in 10 . There it is shown that topologically equivalent foliations are in fact analytically equivalent. This property contrasts with the situation in the real domain, where generic foliations on $\mathbb{R} \mathbb{P}^{2}$ are structurally stable. Later, in [7 X. Gómez-Mont and L. Ortíz-Bobadilla generalized the rigidity theorem of Ilyashenko to many foliations on arbitrary algebraic surfaces admitting an invariant ample curve. We can also cite the results of [9, [13] and 14 on the existence and description of a versal space of equisingular unfoldings. Following the same ideas, it is possible to adapt the proof of Ilyashenko rigidity theorem to many foliations on complex fibred surfaces admitting an invariant fibre.

Before to present the main result we recall some preliminary notions. A holomorphic foliation on a complex surface $S$ is given by a collection of holomorphic vector fields $X_{i}$ on open sets $U_{i}$ which cover $S$ such that if $U_{i} \cap U_{j} \neq \emptyset$ there exists a holomorphic function $g_{i j}: U_{i} \cap U_{j} \rightarrow \mathbb{C}^{*}$ satisfying $X_{i}=g_{i j} X_{j}$. The collection $\left\{g_{i j}^{-1}\right\}$ determines a line bundle $T \mathcal{F}$ on $S$ which is called the tangent bundle of $\mathcal{F}$. The vector fields $X_{i}$ may be singular, but in any case their zeroes (called singularities of $\mathcal{F}$ ) are assumed to be isolated. A singularity $s$ of $X_{i}$ is called reduced if the

Received by the editors April 15, 2005 and, in revised form, June 29, 2005.

2000 Mathematics Subject Classification. Primary 32G34, 34Mxx, 57R30.

Key words and phrases. Singular holomorphic foliation, complex fibred surface, deformation, unfolding, holonomy, rigidity.

(C)2006 American Mathematical Society Reverts to public domain 28 years from publication 
linear part of $X_{i}$ at $s$ has at least one of the two eigenvalues different from zero and moreover its ratio is not a positive rational number. Theses singularities are important because they are the final ones in the reduction process of the singularities of an arbitrary foliation in dimension two. For more details about these notions we refer to 3 .

Theorem A. Let $S$ be a compact complex fibred surface (i.e. admitting a submersion over a complex curve) and let $\mathcal{F}$ be a holomorphic foliation on $S$ having reduced singularities and verifying the following conditions:

(a) There is an invariant fibre $F$ whose holonomy group is rigid and contains a hyperbolic element.

(b) $H^{1}\left(S, \mathcal{O}_{S}(T \mathcal{F})\right)=0$, where $T \mathcal{F}$ is the tangent bundle of the foliation $\mathcal{F}$.

Then, $\mathcal{F}$ is d-rigid, i.e. any topologically trivial holomorphic deformation $\mathcal{F}_{t}$ of $\mathcal{F}$ is also analytically trivial.

Remark 1.1. The rigidity of the holonomy group $G \hookrightarrow \operatorname{Diff}(\mathbb{C}, 0)$ means that if $\psi:(\mathbb{C}, 0) \rightarrow(\mathbb{C}, 0)$ is a germ of homeomorphism such that for any $g \in G$ the composition $\psi \circ g \circ \psi^{-1}$ is an element of $\operatorname{Diff}(\mathbb{C}, 0)$, then $\psi$ is a conformal or anticonformal mapping. In particular, if $\psi$ preserves the orientation, then $\psi$ is a biholomorphism. An element $g$ of $\operatorname{Diff}(\mathbb{C}, 0)$ is called hyperbolic if its linear part $g^{\prime}(0)$ has modulus different from 1 . There are many situations assuring the rigidity of $G$; see [4, 10]. For instance, if $G$ is not abelian and the linear parts of the elements in $G$ form a dense subset of $\mathbb{C}^{*}$, then $G$ is rigid. We point out that these conditions are generically verified for a finite generated subgroup of $\operatorname{Diff}(\mathbb{C}, 0)$ and that they were also required in the earlier works [10, 7]. Another situation implying rigidity is when $G$ is non-solvable (see [15]).

Remark 1.2. We also point out that if $G$ is a rigid subgroup of Diff $(\mathbb{C}, 0)$ containing a hyperbolic element, then $G$ cannot be abelian. Indeed, any abelian subgroup of Diff $(\mathbb{C}, 0)$ containing a hyperbolic element is linearizable, i.e. there is a local coordinate $z$ of $(\mathbb{C}, 0)$ such that each $g \in G$ is of the form $g(z)=\lambda_{g} z$ for some $\lambda_{g} \in \mathbb{C}^{*}$. Morever, the non-conformal homeomorphism $\psi_{c}(z)=z|z|^{c}, c \neq 0$, conjugates $g(z)$ and $h(z)=\mu_{h} z$, where $\mu_{h}=\lambda_{g}\left|\lambda_{g}\right|^{c}$.

We will particularize the above theorem to the case of a ruled surface $\pi: S \rightarrow B$, i.e. when the fibre $F$ is the complex line $\mathbb{C P}^{1}$. A complete study of the coholomogy groups $H^{1}\left(S, \mathcal{O}_{S}(L)\right)$ for any line bundle $L \rightarrow S$ over a ruled surface was done in 8. It follows from that paper that if $\mathcal{F}$ is a holomorphic foliation on $S$ with isolated singularities, then $H^{1}\left(S, \mathcal{O}_{S}(T \mathcal{F})\right)=0$, except when $\mathcal{F}$ belongs to one of the following exceptional cases:

(i) The fibration $p: S \rightarrow B$, which is unique unless for the product $S=$ $\mathbb{C P}^{1} \times \mathbb{C P}^{1}$

(ii) Foliations which are transverse to the fibration outside a finite number of invariant fibres, i.e. Riccati foliations.

(iii) Regular foliations (i.e. without singularities) over a ruled surface whose base $B$ is an elliptic curve $E$. Moreover, if a such a foliation does not belong to one of the preceding two cases, then $S$ is a non-ramified finite quotient of the product $\mathbb{C P}^{1} \times E$. This class includes the turbulent foliations studied by M. Brunella in [2] and [3. 
(iv) Foliations on the first Hirzebruch surface whose tangent line bundle have Chern class $\mathbf{f}-n \mathbf{s}, n \geq 2$, where $\mathbf{f}$ and $\mathbf{s}$ are respectively the Poincaré dual of a fibre and of a holomorphic section of autointersection +1 in $S$. Examples of these types of foliations can be obtained by blowing-up a regular point of any foliation of degree $n \geq 2$ on $\mathbb{C P}^{2}$; see the proof of Proposition 5.3 in [8.

Remark 1.3. Let $\mathcal{F}$ be a holomorphic foliation on a ruled surface admitting an invariant fibre with rigid holonomy group and containing a hyperbolic element. Then $\mathcal{F}$ cannot belong to the classes (i)-(iii) because its holonomy group would be abelian, thus contradicting Remark 1.2, However, there are foliations of degree $n \geq 2$ on $\mathbb{C P}^{2}$ admitting an invariant line $L$ with rigid holonomy group and containing a hyperbolic element; cf. [10. By blowing-up a regular point of $L$ we would obtain a new foliation on the first Hirzebruch surface admitting the strict transform of $L$ as an invariant fibre with the same holonomy group as $L$.

From Theorem $\mathrm{A}$ and the preceding considerations we immediately deduce:

Corollary B. Let $S$ be a ruled surface and let $\mathcal{F}$ be a holomorphic foliation on $S$ with reduced singularities. Assume that $\mathcal{F}$ possesses an invariant fibre $F$ whose holonomy group is rigid and contains a hyperbolic element. If $\mathcal{F}$ does not belong to the exceptional class (iv) described above, then $\mathcal{F}$ is d-rigid.

We can conclude by saying that Riccati foliations form the widest class of nonrigid foliations on ruled surfaces. Hence it would be interesting to determine its analytic moduli space; see [12].

\section{Deformations And Unfoldings}

In order to explain Theorem A, we begin by recalling the difference between topologically trivial deformations and unfoldings and some well-known facts about them. In order to simplify the exposition we restrict ourselves to the case where the parameter space is a disk $\Delta \ni 0$, or more precisely the germ $(\Delta, 0)$. Let $S$ be a complex surface and let $\mathcal{F}$ be a (singular) holomorphic foliation on $S$. A deformation of $\mathcal{F}$ is a deformation $\pi: \mathcal{S} \rightarrow \Delta$ of the underlying surface $S$ and a holomorphic foliation by curves $\mathcal{F}^{\Delta}$ on $\mathcal{S}$ such that:

- the singular set $\Sigma$ of $\mathcal{F}^{\Delta}$ has codimension greater than 1, and none of its irreducible components is contained in a fibre of $\pi$;

- the leaves of $\mathcal{F}^{\Delta}$ are contained in the fibres of $\pi, \pi^{-1}(0)=S$ and $\mathcal{F}_{\mid S}^{\Delta}=\mathcal{F}$.

The deformation $\mathcal{F}^{\Delta}$ is said to be topologically trivial if there exists a homeomorphism $\Phi: \mathcal{S} \rightarrow S \times \Delta$ commuting with the projections over $\Delta$ such that $\Phi^{*}\left(\mathcal{F}^{\text {triv }}\right)=\mathcal{F}^{\Delta}$, i.e. $\Phi$ sends leaves of $\mathcal{F}^{\Delta}$ into leaves of the constant foliation $\mathcal{F}^{\text {triv }}$ on $S \times \Delta$ (whose leaves are $L \times\{t\}$, where $L$ is a leaf of $\mathcal{F}$ and $t \in \Delta$ ). An unfolding of $\mathcal{F}$ is a holomorphic foliation $\mathcal{G}$ of codimension one on the total space $\mathcal{S}$ of some deformation $\pi: \mathcal{S} \rightarrow \Delta$ of $S=\pi^{-1}(0)$ such that the singular set of $\mathcal{G}$ has codimension greater than 1 , none of its irreducible components is contained in a fibre of $\pi$, the leaves of $\mathcal{G}$ are transverse to the fibres of $\pi$ and $\mathcal{G}_{\mid S}=\mathcal{F}$; see [13.

Each unfolding $\mathcal{G}$ of $\mathcal{F}$ determines a deformation in the obvious way: its leaves are the intersections of the leaves of $\mathcal{G}$ with the fibres of $\pi$. Clearly, a priori, an unfolding has a richer structure than a deformation because in the former we have described how the one-dimensional leaves are put together in order to form the 
two-dimensional leaves of the unfolding. In section 4, we recall some known facts about unfoldings of holomorphic foliations. The hypothesis on the singularities and the cohomological assumptions in Theorem A will imply that any unfolding of $\mathcal{F}$ is analytically trivial. Hence, the key step in the proof of Theorem A is a partial converse of the above remark unfolding implies deformation. More concretely, we have the following result in the spirit of Ilyashenko's rigidity theorem, although the proof presented here is inspired by the exposition of 7 .

Theorem 2.1. Let $\mathcal{F}$ be a holomorphic foliation in a complex surface $S$ admitting an invariant curve $C$ such that

(a) the holonomy group of each connected component of $C^{*}=C \backslash \operatorname{Sing}(\mathcal{F})$ is rigid and contains a hyperbolic element;

(b) each leaf $L$ of $\mathcal{F}$ verifies $\bar{L} \cap C^{*} \neq \emptyset$, unless maybe a finite set of closed leaves.

Then every topologically trivial deformation of $\mathcal{F}$ is underlying to an unfolding.

Proof. Let $\mathcal{F}^{\Delta}$ be a topologically trivial deformation of $\mathcal{F}$ on $\pi: \mathcal{S} \rightarrow \Delta$. Let $\Sigma$ be the singular set of $\mathcal{F}^{\Delta}$, which is an analytic set of $\mathcal{S}$ of dimension $\leq 1$. Consider the open subset $\mathcal{S}^{*}=\mathcal{S} \backslash \Sigma$ of $\mathcal{S}$. Since $\mathcal{F}^{\Delta}$ is topologically trivial there exists a homeomorphism $\Phi: \mathcal{S} \rightarrow S \times \Delta$ commuting with the projection over $\Delta$ such that $\Phi^{*}\left(\mathcal{F}^{\text {triv }}\right)=\mathcal{F}^{\Delta}$. Let us consider the topological foliation of codimension one $\mathcal{G}^{\text {top }}=\Phi^{*}(\mathcal{F} \times \Delta)$ on $\mathcal{S}^{*}$ whose leaves are $\Phi^{-1}(L \times \Delta)$, where $L$ is any leaf of $\mathcal{F}$. Note that the deformation induced by the topological unfolding $\mathcal{G}^{\text {top }}$ is just the holomorphic deformation $\mathcal{F}^{\Delta}$. We shall see that $\mathcal{G}^{\text {top }}$ is a holomorphic foliation of codimension one on $\mathcal{S}^{*}$ which will extend holomorphically to the whole $\mathcal{S}$ thanks to Hartogs' theorem, concluding in this way the proof.

Let $\left\{\mathcal{U}_{i}, \varphi_{i}\right\}$ be a system of distinguished maps of $\mathcal{F}^{\Delta}$ restricted to $\mathcal{S}^{*}$, i.e. $\mathcal{U}_{i}$ are open sets of $\mathcal{S}^{*}$ and $\varphi_{i}: \mathcal{U}_{i} \rightarrow V_{i} \subset \mathbb{C}^{2}$ are submersions defining $\mathcal{F}_{\mid \mathcal{U}_{i}}^{\Delta}$. Let us define the local (topological) foliation $\mathcal{G}_{i}=\left(\varphi_{i}\right)_{*}\left(\mathcal{G}^{\text {top }}\right)$ on $V_{i}$.

Since $\mathcal{F}^{\Delta}$ is topologically trivial, without loss of generality we can assume that $\mathcal{U}_{i} \cong U_{i} \times \Delta, V_{i}=V_{i}^{0} \times \Delta$ and $\varphi_{i}(p, t)=\left(\varphi_{i}^{t}(p), t\right)$. On the other hand, the holomorphy of $\mathcal{G}^{\text {top }}$ is a local property. Hence, it suffices to show that $\mathcal{G}_{i}$ is holomorphic because $\mathcal{G}_{\mid \mathcal{U}_{i}}^{\text {top }}=\varphi_{i}^{*} \mathcal{G}_{i}$. In order to show this, we proceed in four steps:

(I) If $\mathcal{U}_{i} \cap C \neq \emptyset$, then the leaf $C_{i}$ of $\mathcal{G}_{i}$ corresponding to $\Phi^{-1}(C \times \Delta)$ is holomorphic. To see this we will use the existence of a hyperbolic element in the holonomy group $G$ of the corresponding connected component of $C^{*}$.

(II) Using the rigidity of $G$ we will see that $\mathcal{G}_{i}$ is holomorphic in a neighborhood of $C_{i}$.

(III) Hypothesis (b) implies that $\mathcal{G}_{i}$ is holomorphic outside a finite set of closed leaves.

(IV) Finally, using a variant of the Riemann extension theorem we will conclude that $\mathcal{G}_{i}$ is holomorphic in the whole open set $V_{i} \subset \mathbb{C}^{2}$.

(I) Let $\Sigma_{i}=\Sigma_{i}^{0} \times \Delta$ be a transverse section to $\mathcal{F}^{\Delta}$ in $\mathcal{U}_{i}$. In fact, we could identify $\Sigma_{i}$ with $V_{i}$ through the restriction to $\Sigma_{i}$ of the map $\varphi_{i}$. Consider $p_{i} \in$ $C \cap \Sigma_{i}$ and $\psi_{i}:\left(\Sigma_{i}, p_{i}\right) \rightarrow\left(\Sigma_{i}, p_{i}\right)$ a hyperbolic transformation in the holonomy group of the connected component of $C^{*}$ which contain the point $p_{i}$. We can write $\psi_{i}(z, t)=\left(\psi_{i}^{t}(z), t\right)$ if $z \in \Sigma_{i}^{0} \subset \mathbb{C}$ and $t \in \Delta$. Let $(z, t)$ be local coordinates in $\Sigma_{i}$ 
such that $p_{i}=(0,0)$. The hyperbolicity of $\psi_{i}$ means that the inequality

$$
\left|\frac{\partial}{\partial z}\right|_{z=0} \psi_{i}^{t}(z) \mid<1
$$

holds for $t=0$. Hence, if $\Delta$ is small enough, then (1) also holds for every $t \in \Delta$. Let $Z_{i}$ be the fixed set of $\psi_{i}$. Thanks to (1), we can apply the Implicit Function Theorem to conclude that $Z_{i}$ is a regular curve in $V_{i}$ which is locally the graph of a holomorphic function $z_{i}: \Delta \rightarrow \Sigma_{i}^{0} \subset \mathbb{C}$ with $z_{i}(0)=0$. It is easy to see that $C_{i}=\varphi_{i}\left(Z_{i}\right) \subset V_{i}$ and consequently, the leaf $C_{i}$ is holomorphic.

(II) Since $z_{i}: \Delta \rightarrow \Sigma_{i}^{0}$ is holomorphic, we can change the coordinates in order to have $z_{i} \equiv 0$ and consequently $C_{i}=\varphi_{i}(\{0\} \times \Delta)$. Let us consider the local homeomorphism $\bar{\phi}_{i}^{t}:(\mathbb{C}, 0) \rightarrow(\mathbb{C}, 0)$ which makes the following diagram commutative:

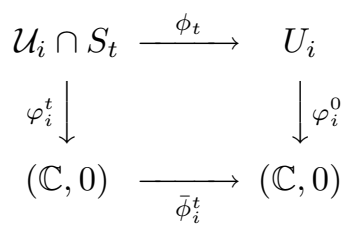

where $S_{t}=\pi^{-1}(t)$ and $\phi_{t}=\Phi_{\mid S_{t}}$. By construction, $\bar{\phi}_{i}^{t}$ is a topological conjugation of the holonomies of $C_{t}=\phi_{t}^{-1}(C)$ and $C$ with respect to the foliations by curves $\mathcal{F}_{t}=\mathcal{F}_{\mid S_{t}}^{\Delta}$ and $\mathcal{F}$. Hypothesis (a) implies that $\bar{\phi}_{i}^{t}(z)$ is holomorphic with respect to the coordinate $z$. We shall see that $\bar{\phi}_{i}^{t}$ is also holomorphic with respect to the coordinate $t \in \Delta$. To prove this, we consider an analytic family of contractive holonomy diffeomorphisms $\psi_{i}^{t}$ conjugated to $\psi_{i}^{0}$ by $\bar{\phi}_{i}^{t}$. Thanks to the Schroeder linearization theorem for analytic families of diffeomorphism of $(\mathbb{C}, 0)$ (cf. for instance [7]), there is an analytic family of linearizing coordinates $\zeta_{t}$ such that $\bar{\phi}_{i}^{t}\left(\zeta_{t}\right)=\sigma(t) \zeta_{0}$ for some continuous function $\sigma: \Delta \rightarrow \mathbb{C}^{*}$.

Since the holonomy group $G$ is non-abelian (cf. Remark 1.2) there exists an element $g \in G$, such that $g\left(\zeta_{0}\right)=\sum a_{k} \zeta_{0}^{k}$ with $a_{k} \neq 0$ for some $k>1$. The holonomy element

$$
\left(\bar{\phi}_{i}^{t}\right)^{-1} \circ g \circ \bar{\phi}_{i}^{t}=\sum_{k=1}^{\infty} \frac{a_{k}}{\sigma(t)^{k-1}} \zeta_{t}^{k}
$$

depends analytically on $t$, therefore we obtain that $\sigma$ is holomorphic and consequently $\bar{\phi}_{i}^{t}$ also is holomorphic with respect to $t$. It is easy to see that $\mathcal{G}_{i}$ is defined in a neighborhood of $C_{i}$ by the level sets of the map $(z, t) \mapsto \bar{\phi}_{i}^{t}(z)$.

(III) Since the hypothesis (b) is purely topological, it is also verified by $\left(S_{t}, \mathcal{F}_{t}, C_{t}\right)$. Therefore, for every $j$, the set $V_{j}^{*}$ of points $\varphi_{j}(p) \in V_{j}$, such that the leaf of $\mathcal{F}^{\Delta}$ through $p$ is adherent to $\mathcal{C}=\Phi^{-1}(C \times \Delta)=\bigcup_{t \in \Delta} C_{t}$ in regular points, is an open set whose complementary is a finite union of plaques of $\mathcal{G}_{j}$. Point (II) implies that $\mathcal{C}$ is a holomorphic leaf of the topological foliation $\mathcal{G}^{\text {top }}$ having a neighborhood where it is holomorphic. Consider $(z, t) \in V_{j}^{*}$ and $p \in \mathcal{U}_{j}$ such that $\varphi_{j}(p)=(z, t)$. Since the leaf $L_{t}$ of $\mathcal{F}^{\Delta}$ through $p$ accumulates $C_{t}$ in regular points, there exists $q \in L_{t} \cap \mathcal{U}_{i}$ close enough to $C_{t}$ such that $\mathcal{G}_{i}$ is holomorphic in a neighborhood of $\varphi_{i}(q) \in V_{i}$. Let $\gamma$ be a path inside $L_{t}$ joining the points $p$ and $q$, and consider the holonomy transformation $\psi_{\gamma}: V_{j} \rightarrow V_{i}$ of the foliation $\mathcal{F}^{\Delta}$ associated to $\gamma$. It is easy to see that $\mathcal{G}_{j}=\psi_{\gamma}^{*} \mathcal{G}_{i}$ and therefore, $\mathcal{G}_{j}$ is holomorphic in a neighborhood of $(z, t)$. Hence $\mathcal{G}_{j}$ is holomorphic in $V_{j}^{*}$. 
(IV) Finally, we will prove that $\mathcal{G}_{j}$ is holomorphic in the whole $V_{j}$. As we have seen, $\mathcal{G}_{j}$ can be defined by a continuous function $f: V_{j} \rightarrow \mathbb{C}$ (sending $(z, t)$ into $\left.f(z, t)=\bar{\phi}_{i}^{t}(z)\right)$ which is holomorphic in $V_{j}^{*}$. By construction, there exist positive constants $r_{2}>r_{1}>0$ such that $K:=\left\{z \in \Sigma_{i}^{0}: r_{1}<|z|<r_{2}\right\} \times \Delta \subset V_{j}^{*}$. For any $r \in\left(r_{1}, r_{2}\right)$, the function

$$
\tilde{f}(z, t)=\frac{1}{2 i \pi} \int_{|\zeta|=r} \frac{f(\zeta, t)}{\zeta-z} d \zeta
$$

is holomorphic in $z$ and coincides with $f$ on $V_{j}^{*}$. On the other hand,

$$
\frac{\partial \tilde{f}}{\partial \bar{t}}(z, t)=\frac{1}{2 i \pi} \int_{|\zeta|=r} \frac{\frac{\partial f}{\partial t}(\zeta, t)}{\zeta-z} d \zeta=0
$$

because $f$ is holomorphic in $K \subset V_{j}^{*}$. It follows from Osgood's lemma that $\tilde{f}$ is holomorphic in $V_{j}$. Since $\tilde{f}=f$ in $V_{j}^{*}$ and $f$ is continuous in $V_{j}$, it follows from the Riemann extension theorem that $f=\tilde{f}$ is holomorphic in the whole $V_{j}$.

Remark 2.2. Thanks to the rigidity of the non-solvable dynamics in Diff( $\mathbb{C}, 0)$ and the density of contractive fixed points (see [15, 1]), we can substitute the hypothesis (a) in Theorem 2.1 by

$\left(\mathrm{a}^{\prime}\right)$ the holonomy group of each connected component of $C^{*}=C \backslash \operatorname{Sing}(\mathcal{F})$ is non-solvable.

\section{Complex fibred surfaces}

For a complex fibred surface $S$ we mean a complex surface admitting an analytic locally trivial fibration $p: S \rightarrow B$ over a complex curve $B$. We will use the following notion and result of R. Gérard and A. Sec in [5, which were originally inspired by the work of P. Painlevé (cf. [16]).

Definition 3.1 (Gerard-Sec). A foliation $\mathcal{F}$ on $S$ is said to be simple with respect to a map $p: S \rightarrow B$ if and only if each point $s \in S$ has a $\mathcal{F}$-distinguished neighborhood $U$ such that the plaque through $s$ meets $p^{-1}(p(s))$ only in the point $s$.

Remark 3.2. If $S$ is a compact surface and $p: S \rightarrow B$ is a submersion it follows from Ehresmann's Lemma that $p$ is a locally trivial fibration. In this situation, assume that $\mathcal{F}$ is a (singular) holomorphic foliation on $S$, and let us denote by $\Sigma$ the union of the singular set of $\mathcal{F}$ and the fibres of $p$ invariant by $\mathcal{F}$. Then it is easy to see that $\mathcal{F}_{\mid S^{*}}$ is simple with respect to $p_{\mid S^{*}}: S^{*} \rightarrow B^{*}$, where $B^{*}=B \backslash p(\Sigma)$ and $S^{*}=p^{-1}\left(B^{*}\right)$.

Theorem 3.3 (Gerard-Sec). If $p: S \rightarrow B$ is an analytic locally trivial fibration and $\mathcal{F}$ is a simple foliation with respect to $p$, then for each path $\gamma:[0,1] \rightarrow B$ and for each point $s \in p^{-1}(\gamma(0))$ there exists a lifting $\tilde{\gamma}$ of $\gamma$ to the leaf of $\mathcal{F}$ passing through $s=\tilde{\gamma}(0)$. Moreover, the number of liftings is bounded by an integer depending only on the point $s$.

We will also need a theorem of E. Ghys generalizing to the non-algebraic case the well-known result of J.-P. Jouanolou concerning the finiteness of closed leaves of holomorphic foliations; see [11, 6]. 
Theorem 3.4 (Ghys). If $\mathcal{F}$ is a codimension one (possibly singular) holomorphic foliation on a compact, connected complex manifold, then $\mathcal{F}$ has only a finite number of closed leaves except when $\mathcal{F}$ admits a meromorphic first integral, in which case all leaves are closed.

Now, we proceed to continue with the following step of the proof of Theorem A.

Proposition 3.5. Under the hypothesis of Theorem A, if we put $C=F$, then the hypotheses of Theorem 2.1 hold.

Proof. First of all, we point out that if a leaf $L$ of $\mathcal{F}$ meets all the fibres of $p$ in a finite number of points, then $L$ is compact. Indeed, the restriction of $p: S \rightarrow B$ to $L$ is finite and hence is a proper map. Second, we note that the existence of a meromorphic first integral of $\mathcal{F}$ in $S$ would imply that the holonomy group of each leaf is finite. This would contradict the hypothesis that the holonomy group of $F$ contains a hyperbolic element because it is of infinite order. From Theorem 3.4 we deduce that there is a finite number of compact leaves of $\mathcal{F}$.

Let $L$ be a non-compact leaf of $\mathcal{F}$, i.e. $\bar{L} \backslash L \not \subset \operatorname{Sing}(\mathcal{F})$. Using the notations of Remark 3.2, we fix a point $b_{0}$ in $B^{*}$ and a path $\gamma:[0,1) \rightarrow B^{*}$ such that $\lim _{t \rightarrow 1} \gamma(t)$ exists and is equal to $b_{1}=p(F)$. For each $0<t<1$ and for each point $s$ of $L \cap p^{-1}\left(b_{0}\right)$, we consider the lift $\tilde{\gamma}_{t}$ of $\gamma$ restricted to $[0, t]$ to the leaf $L$ such that $\tilde{\gamma}_{t}(0)=s$. The compactness of $S$ implies that the set $A(\gamma) \subset F$ of accumulation points of $\tilde{\gamma}_{t}$, when $t$ tends to 1 and $s$ ranges $L \cap p^{-1}\left(b_{0}\right)$, is non-empty. Since all the singularities of $\mathcal{F}$ are reduced (in particular those lying on $F$ ), $A(\gamma)$ must necessarily contain some regular point of $F$.

\section{TRIVIAlity of The VERSAL EQUisingular UNFOLDING}

This section contains the last step of the proof of Theorem A, namely unfolding implies analytically trivial. This will be consequence of two facts which follow from the hypothesis assumed on $\mathcal{F}$ in Theorem A:

(A) Every unfolding of $\mathcal{F}$ is equisingular.

(B) Every equisingular unfolding of $\mathcal{F}$ is analytically trivial.

To show this we shall recall some facts about equisingular and versal unfoldings.

(A) Let $\mathcal{G}$ be a holomorphic unfolding (over a parameter space $P$ of arbitrary dimension) of a singular foliation $\mathcal{F}$ on a complex surface $S$. Roughly speaking, $\mathcal{G}$ is equisingular if it admits a reduction of singularities $\varpi: \widetilde{\mathcal{S}} \rightarrow \mathcal{S}$ whose restriction to $\varpi^{-1}\left(S_{t}\right)$ gives us the reduction of $\mathcal{F}_{t}=\mathcal{F}_{\mid S_{t}}$, where $S_{t}=\pi^{-1}(t)$ and $\pi: \mathcal{S} \rightarrow P$ is the underlying ambient deformation of the unfolding $\mathcal{G}$. We refer to [13, 14] for a proper definition. It is well known that every local unfolding of a reduced singularity is analytically trivial (see for instance Lemme 1.1.5 of [13]). This is also the case for a non-reduced singularity of Milnor number equal to one and nonvanishing trace (Lemma 5.6 of [9]). This fact implies in particular that every local unfolding of a reduced singularity is equisingular. Since equisingularity is a local property we conclude that, for a global foliation $\mathcal{F}$ with reduced singularities, every unfolding is equisingular.

(B) An unfolding $\mathcal{G}^{\text {ver }}$ of $\mathcal{F}$ over $P^{\text {ver }}$ is called versal if it contains any other unfolding $\mathcal{G}$ of $\mathcal{F}$ over $P$. More precisely, if there exists a map $\lambda_{P}: P \rightarrow P^{\text {ver }}$ so 
that we can complete the following pull-back diagram:

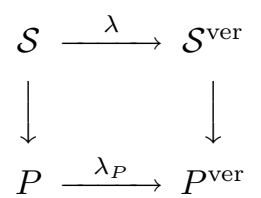

i.e., $\mathcal{S} \cong \lambda_{P}^{*} \mathcal{S}^{\text {ver }}$ and $\mathcal{G} \cong \lambda^{*} \mathcal{G}^{\text {ver }}$. In [14], a versal equisingular unfolding is constructed for every foliation $\mathcal{F}, P^{\text {ver }}$ being a (possibly singular) analytic space. Under some cohomological assumptions it is shown that $P^{\mathrm{ver}}$ is smooth and naturally identified with the product of the parameter spaces $P_{i}^{\text {loc }}$ (see also [13]) corresponding to the local equisingular versal unfoldings of the germs of $\mathcal{F}$ at each singular point $s_{i}$ of $\mathcal{F}, i=1, \ldots, k$.

Theorem 4.1 (Mattei-Nicolau). Let $\mathcal{F}$ be a foliation by curves with isolated singularities in a compact surface $S$. Assume that $H^{1}\left(S, \mathcal{O}_{S}(T \mathcal{F})\right)=0$. Then the differential at 0 of the localization map

$$
\chi: P^{\mathrm{ver}} \longrightarrow \prod_{i=1}^{k} P_{i}^{\mathrm{loc}}
$$

is an isomorphism. Moreover, if $H^{2}\left(S, \mathcal{O}_{S}(T \mathcal{F})\right)$ also vanishes, then $P^{\mathrm{ver}}$ is smooth and $\chi$ is an isomorphism.

Indeed, as we have already mentioned, if all the singularities of $\mathcal{F}$ are reduced, then $P_{i}^{\text {loc }}=0$. If, in addition, $H^{1}\left(S, \mathcal{O}_{S}(T \mathcal{F})\right)=0$, then we do not need the vanishing of the second cohomology group of $T \mathcal{F}$ in order to conclude that $P^{\text {ver }}=0$. Consequently, every equisingular unfolding of $\mathcal{F}$ is analytically trivial. We also point out that in [9] is proved the triviality of the equisingular versal unfolding of $\mathcal{F}$ provided that all its singularities have Milnor number equal to 1 and nonvanishing trace.

\section{REFERENCES}

[1] M. Belliart, I. Liousse, and F. Loray. Sur l'existence de points fixes attractifs pour les sous-groupes de Aut(C, 0). C.R. Acad. Sci. Paris, Série I, 324:443-446, 1997. MR.1440964 (98c:58134)

[2] Marco Brunella. Feuilletages holomorphes sur les surfaces complexes compactes. Ann. Sci. École Norm. Sup. (4), 30(5):569-594, 1997. MR1474805 (98i:32051)

[3] Marco Brunella. Birational geometry of foliations. Monografías de Matemática. Instituto de Matemática Pura e Aplicada (IMPA), Rio de Janeiro, 2000. MR.1948251 (2004g:14018)

[4] Dominique Cerveau and Paulo Sad. Problèmes de modules pour les formes différentielles singulières dans le plan complexe. Comment. Math. Helv., 61(2):222-253, 1986. MR0856088 (88f:58124)

[5] Raymond Gérard and Antoinette Sec. Feuilletages de Painlevé. Bull. Soc. Math. France, 100:47-72, 1972. MR0306552(46:5675)

[6] Étienne Ghys. À propos d'un théorème de J.-P. Jouanolou concernant les feuilles fermées des feuilletages holomorphes. Rend. Circ. Mat. Palermo (2), 49(1):175-180, 2000. MR1753461 (2001i:32048)

[7] X. Gómez-Mont and L. Ortíz-Bobadilla. Sistemas dinámicos holomorfos en superficies. Sociedad Matemática Mexicana, México City, 1989. MR1304495 (95j:32046)

[8] Xavier Gómez-Mont. Holomorphic foliations in ruled surfaces. Trans. Amer. Math. Soc., 312(1):179-201, 1989. MR0983870 (91e:32027)

[9] Xavier Gómez-Mont. Unfoldings of holomorphic foliations. Publ. Mat., 33(3):501-515, 1989. MR:1038486 (91d:32026) 
[10] Yu. S. Ilyashenko. Topology of phase portraits of analytic differential equations on a complex projective plane. Trudy Sem. Petrovsk., (4):83-136, 1978. MR0524528 (84k:58164)

[11] J. P. Jouanolou. Hypersurfaces solutions d'une équation de Pfaff analytique. Math. Ann., 232(3):239-245, 1978. MR0481129 (58:1274)

[12] David Marín and Marcel Nicolau. Riccati foliations on ruled surfaces. In preparation, 2005.

[13] Jean-François Mattei. Modules de feuilletages holomorphes singuliers. I. Équisingularité. Invent. Math., 103(2):297-325, 1991. MR1085109 (92f:32056)

[14] Jean-François Mattei and Marcel Nicolau. Equisingular unfoldings of foliations by curves. Astérisque, 222(6):285-302, 1994. Complex analytic methods in dynamical systems (Rio de Janeiro, 1992). MR1285392 (96c:32040)

[15] Isao Nakai. Separatrices for nonsolvable dynamics on (C,0). Ann. Inst. Fourier (Grenoble), 44(2):569-599, 1994. MR1296744 (95j:58124)

[16] M. P. Painlevé. Leçons sur la théorie analytique des équations différentielles. Librairie scientifique A. Hermann, 1897. Leçons professées a Stockholm, 1895.

Departament de Matemàtiques, Universitat Autònoma de Barcelona, E-08193 Bellaterra, Barcelona, Spain

E-mail address: davidmp@mat.uab.es 